\title{
Ueber die Endergebnisse der vaginalen Total- exstirpation wegen Carcinom an der Königlichen Frauenklinik zu Dresden.
}

\author{
Von \\ Dr. Eduard Leisse, \\ erstem Assistenter.
}

In Folge der Kritik, die in jüngster Zeit an den unter obigem Titel von hier ausgegangenen Arbeiten geübt worden ist, hat sich Herr Prof. Leopold veranlasst gesehen, in eine erneute Prüfung der bisher erzielten Ergebnisse einzutreten. Es wurde dieserhalb an alle noch Lebenden bezw. für noch lebend Gehaltenen die Aufforderung gesandt, sich persönlich zur Nachuntersuchung vorzustellen, oder, falls das nicht möglich sein sollte, wenigstens Auskunft über jhr Befinden zu geben, und zwar erstreckte sich diese Aufforderung an alle Diejenigen, deren Operation bei Abfassung dieser Arbeit zwei Jahre oder mehr hinter uns lag. Die auf diese Weise erhaltenen Ergebnisse sind in folgender Tabelle (S. 262-264) niedergelegt, welche zur Erleichterung des Vergleiches mit früheren Arbeiten (dieses Archiv, Bd. XXX und XXXVI, and Münchener medicinische Wochenschrift Nr. 46, Jahrg. 1890) überhaupt alle bis Anfang Mai 1889 in hiesiger Anstalt wegen Carcinom ausgeführten vaginalen Totalexstirpationen zusammenstellt.

Um nun die in dieser Tabelle niedergelegten Ergebnisse kurz zusammenzufassen, so sind von 80 Jänger als zwei Jahre Operirten noch am Leben . . . . $45=56,25$ Proe.
gestorben sind insgesammt . $35=43,75 "$

Die Todesfälle häufen sich gegen das Ende dẹ Tabelle. Es erklärt sich diese Erscheinung ungezwungen daraus, dass mit fortschreitender Uebung auch technisch schwierigere Fälle der Radicaloperation unterworfen wurden, die also für den Dauererfolg viel weniger günstige Aussichten bieten mussten. 
262 Leisse, Jeber die Enảergebnisse der vaginalen Totalexstirpation

\begin{tabular}{|c|c|c|c|c|}
\hline$\ddot{z}$ & Touma & Tag der & Nach brieflich & er Aufforderung erfolgte \\
\hline 焉 & & Operation & Antwort & Vorstellung \\
\hline 1 & Fr. Kr. & 11. Oct. 83 . & - & $\begin{array}{l}\text { 27. Jan. 91: Kein Rückfall. } \\
\text { Glatte Narbe. }\end{array}$ \\
\hline 2 & Fr. $\mathrm{K}$. & 12. Oct. 83 . & 一 & $\begin{array}{l}\text { 18. April 91: Kein Rückfall. } \\
\text { Glatte Narbe. }\end{array}$ \\
\hline 3 & Fr. R. & 11. Sept. 84 . & - & $\begin{array}{l}\text { 9. Febr. 91: Kein Rückfall. } \\
\text { Glatte Narbe. }\end{array}$ \\
\hline 4 & Fr. M. †. & 29. Sept. 84 . & - & - \\
\hline 5 & Fr. R. & 30. Sept. 84 & $\begin{array}{l}\text { 18. Jan. } 91 \text { : Befinden } \\
\text { gut. }\end{array}$ & $\begin{array}{l}\text { 4. Mai 91: Kein Rückfall. } \\
\text { Glatte Narbe. }\end{array}$ \\
\hline 6 & $\begin{array}{l}\text { Fr. M. } \dagger \\
\text { Psychose. }\end{array}$ & 2. Dec. 84 . & - & - \\
\hline 7 & Fr. Schm. $\dagger$. & 11. Dec. 84 . & - & - \\
\hline 8 & Fr. Sudf. & 27. Jan. 85 . & - & $\begin{array}{l}\text { 12. Jau. 91: Kein Rückfall. } \\
\text { Glatte Narbe. }\end{array}$ \\
\hline 9 & Fr. Hipp. & 30. Jan. 85 & $\begin{array}{l}\text { 25. März 91: Befin- } \\
\text { den sehr gut. }\end{array}$ & - \\
\hline 10 & $\begin{array}{l}\text { Fr. G. } † . \\
\text { Sepsis. }\end{array}$ & 10. Mai 85 . & - & - \\
\hline 11 & Fr. Fischer. & 4. Juni 85 . & - & $\begin{array}{l}\text { 31. März 91: Kein Rückfall. } \\
\text { Glatte Narbe. }\end{array}$ \\
\hline 12 & $\begin{array}{l}\text { W. N. }{ }^{\dagger} . \\
\text { Erschöptung. }\end{array}$ & 13. Juni 85 . & - & - \\
\hline 13 & F. W. & 18. Juni 85 . & - & $\begin{array}{l}\text { 11. April 91: Vollständiges } \\
\text { Wohlbefinden. (Hausarzt.) }\end{array}$ \\
\hline 14 & Fr. Bl. & 3. Juli 85. & - & $\begin{array}{l}\text { 7. April 91: Kein Rückfall. } \\
\text { Glatte Narbe. }\end{array}$ \\
\hline 15 & Fr. F. & 11. Juli 85 . & - & $\begin{array}{l}\text { 2. April 91: Kein Rückfall. } \\
\text { Glatte Narbe. }\end{array}$ \\
\hline 16 & $\begin{array}{l}\text { Fr. Rost. } \dagger . \\
\text { Phthisis. }\end{array}$ & 24. Juli 85 . & - & - \\
\hline 17 & $\begin{array}{l}\text { Fr. Erd. } † . \\
\text { Psychose. }\end{array}$ & 20. Sept. 85 & - & $=0$ \\
\hline 18 & Fr. Sieber. & 3. Nov. 85 . & $\cdots$ & $\begin{array}{l}\text { 27. Jan. 91: Kein Rückfall. } \\
\text { Glatte Narbe. }\end{array}$ \\
\hline 19 & W. Schütze.†. & 10. Nov. 85. & - & - \\
\hline 20 & Fr. Gl. & 19. Nov. 85 . & - & $\begin{array}{l}\text { 24. März 91: Kein Rückfall. } \\
\text { Glatte Narbe. }\end{array}$ \\
\hline 21 & Fr. Fr. $\dagger$. & 26. Nov. 85 . & - & - \\
\hline 22 & Fr. H. †. & 6. Jan. 86 . & - & - \\
\hline 23 & Fr. Str. & 19. Jan. 86 . & $\begin{array}{l}\text { 15. März 91: Befinden } \\
\text { sehr gut nach Aussage } \\
\text { von Mann u. Tochter. }\end{array}$ & - \\
\hline 24 & & 26. Jan. 86 . & $\begin{array}{l}\text { 12. Jan. 91: Befinden } \\
\text { z. grösst. Zufriedenheit. }\end{array}$ & 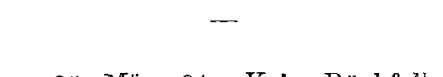 \\
\hline 25 & Fr. Oe. & 4. Febr. 86 . & - & $\begin{array}{l}\text { 25. März 91: Kein Rückfall. } \\
\text { Glatte Narbe. }\end{array}$ \\
\hline 26 & Fr. K. & 5. Felir. 86 & - & $\begin{array}{l}\text { 9. Febr. 91: Kein Rückfall. } \\
\text { Glatte Narbe. }\end{array}$ \\
\hline
\end{tabular}




\begin{tabular}{|c|c|c|c|c|}
\hline i & & Tag der & Nach brieflich & er Aufforderung erfolgte \\
\hline$\stackrel{2}{ت}$ & & Oper & Antwort & Vorstellung \\
\hline 27 & Fr. Th. & 2. März 86 . & - & $\begin{array}{l}\text { 27. April } 91 \text { : Kein Rückfall. } \\
\text { Glatte Narbe. }\end{array}$ \\
\hline 28 & Fr. L. $\dagger$. & 6. März 86. & - & $\rightarrow$ \\
\hline 29 & Fr. Zi. †. & 27. März 86. & - & - \\
\hline 30 & Fr. Hemp. & 30. März 86. & $\begin{array}{l}\text { 25. März 91 Antwort der } \\
\text { Fran: Befinde micl stets } \\
\text { unwohl. - } 25 \text {. April } 91 \\
\text { Antwort des Arztes: Nach } \\
\text { Angabe und Aussehen ge- } \\
\text { sund. }\end{array}$ & $\begin{array}{l}\text { 30. April 91: Kein Rückfall. } \\
\text { Feine Blasenscheidenfistel (siehe } \\
\text { Archiv f. Gyn., Bd. XXX) seit } \\
\text { der Operation. }\end{array}$ \\
\hline 31 & Fr. Berth. & 21. Mai 86 . & $\begin{array}{l}\text { 14. Jan. 91: Allge- } \\
\text { meinbefinden zufrieden- } \\
\text { stellend. }\end{array}$ & $\begin{array}{l}\text { 28. April 91: Kräfte schwach. } \\
\text { Lungenkatarrh. Kein Rückfäll. } \\
\text { Glatte Narbe. }\end{array}$ \\
\hline 32 & Fr. Behr. & 30. Juni 86 . & - & $\begin{array}{l}\text { 23. April 91: Kein Rückfall. } \\
\text { Glatte Narbe. }\end{array}$ \\
\hline 33 & Fr. Besser. & 2. Juli 86 . & - & $\begin{array}{l}\text { 3. März 91: Kein Rückfall. } \\
\text { Glatte Narbe. }\end{array}$ \\
\hline 34 & Fr. Not. $\dagger$. & 24. Juli 86 . & - & - \\
\hline 35 & Fr. Kummer. & 14. Aug. 86 . & $\begin{array}{l}\text { 14. Febr. 91: Voll- } \\
\text { ständiges Wohlbefinden. }\end{array}$ & - \\
\hline 36 & Er. Schmidt. $\dagger$. & 16. Sept. 86 . & - & 一 \\
\hline 37 & Fr. KI. & 15. Oct. 86 . & $\begin{array}{l}\text { 15. März 91: Nach } \\
\text { Bericht d. Mannes voll- } \\
\text { ständiges Wohlbefinden. }\end{array}$ & - \\
\hline 38 & Fr. Arlt. & 22. Oct. 86 . & - & $\begin{array}{l}\text { 4. April 91: Kein Rückfall. } \\
\text { Glatte Narbe. }\end{array}$ \\
\hline 39 & $\begin{array}{l}\text { Fr. Rasch. } \dagger \text {. } \\
\text { Darmverschluss. }\end{array}$ & 4. Jan. 87 . & - & - \\
\hline 40 & Fr. Böhm. †. & 2. Febr. 87. & - & - \\
\hline 41 & Fr. Ruick. & 2. März 87 . & - & $\begin{array}{l}\text { 2. April 91: Kein Rückfall. } \\
\text { Glatte Narbe. }\end{array}$ \\
\hline 42 & Fr. Müller. $\dagger^{\circ}$ & 17. März 87. & - & - \\
\hline 43 & Fr. Hi. & 24. Mai 87. & - & $\begin{array}{l}\text { 20. April 91: Kein Rückfall. } \\
\text { Glatte Narbe. }\end{array}$ \\
\hline 44 & Hens. $\dagger$ & 21. Juni 87 . & - & - \\
\hline 45 & Fr. Fred. & 28. Juni 87. & - & $\begin{array}{l}\text { 6. April 91: Kein Rückfall. } \\
\text { Glatte Narbe. }\end{array}$ \\
\hline 46 & Fr. Her. & 26. Juli 87 . & - & $\begin{array}{l}\text { 12. Jan. 91: Kein Rückfall. } \\
\text { Glatte Narbe. }\end{array}$ \\
\hline 47 & Fr. Lö. †. & 6. Aug. 87. & - & - \\
\hline 48 & Fr. R. & 22. Aug. 87. & - & $\begin{array}{l}\text { 17. Febr. } 91 \text { : Kein Rückfall } \\
\text { Glatte Narbe. }\end{array}$ \\
\hline 49 & $\begin{array}{l}\text { Fr. Jh. } \dagger \text {. } \\
\text { Peritonitis. }\end{array}$ & 27. Aug. 87. & -- & - \\
\hline 50 & Fr. Wi. & 29. Aug. 87 . & - & $\begin{array}{l}\text { 26. Jan. 91: Ungestörtes Be- } \\
\text { finden. (Hausarzt.) }\end{array}$ \\
\hline 51 & Fr. Zi. & 28. Oct. 87 . & - & $\begin{array}{l}\text { 18. Jan. 91: Kein Rückfall. } \\
\text { Gutes Befinden. (Dr. Korn in } \\
\text { Dresden.) }\end{array}$ \\
\hline
\end{tabular}


264 Leisse, Ueber die Endergebnisse der vaginalen Totalexstirpation

\begin{tabular}{|c|c|c|c|c|}
\hline 至 & \multirow{2}{*}{ Namen } & \multirow{2}{*}{$\begin{array}{l}\text { Tag der } \\
\text { Operation }\end{array}$} & \multicolumn{2}{|c|}{ Nach brieflicher Aufforderung erfolgte } \\
\hline 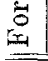 & & & Antwort & Vorstellung \\
\hline 52 & Fr. Schn. $\dagger$. & 29. Nov. 87. & - & - \\
\hline 53 & Fr. Mü. †. & 14. Dec. 87 . & - & - \\
\hline 54 & $\begin{array}{l}\text { Fr. Wich. } t \text {. } \\
\text { Herzfehler. }\end{array}$ & 16. Dec. 87 . & - & - \\
\hline 55 & Fr. Neu. $\dagger$. & 7. Febr. 88 & - & - \\
\hline 56 & Fr. Rieg. & 9. Febr. 88 . & - & $\begin{array}{l}\text { 23. Jan. 91: Kein Rückfall. } \\
\text { Glatte Narbe. }\end{array}$ \\
\hline 57 & Fr. Mie. & 24. Febr. 88 . & $\begin{array}{l}\text { 23. April 91: Unge- } \\
\text { störtes Wohlbefinden. }\end{array}$ & . \\
\hline 58 & Fr. Neub. & 14. März 88. & $\begin{array}{l}\text { 13. Jan. 91: Yoll- } \\
\text { stündig. Wohlbefinden. }\end{array}$ & - \\
\hline 59 & $\mathrm{Fl}_{\mathrm{I}}$. Kot. & 11. April 88 & $\begin{array}{l}\text { 12. Jan. 91: Voll- } \\
\text { ständig. Wohlbefinden. }\end{array}$ & $\begin{array}{l}\text { 24. April 91: Kein Rückfall. } \\
\text { Glatte Narbe. }\end{array}$ \\
\hline 60 & Fr. Wetz. & 29. Mai 88. & - & $\begin{array}{l}\text { 31. März 91: Kein Rückfall. } \\
\text { Glatte Narbe. }\end{array}$ \\
\hline 61 & Fr. Gu. $†$ & 11. Juni 88 . & - & - \\
\hline 62 & Fr. R. & 12. Juli 88 . & - & $\begin{array}{l}\text { 15. Jan. 91: Kein Rückfall. } \\
\text { Glatte Narbe. }\end{array}$ \\
\hline 63 & Fr. Dier. $\dagger$. & 28. Juli 88 . & - & - \\
\hline 64 & Fr. Lel. & 3. Aug. 88 . & - & $\begin{array}{l}\text { 23. Febr. 91: Kein Rückfall. } \\
\text { Glatte Narbe. }\end{array}$ \\
\hline 65 & Fr. Forb. $†$. & 23. Oct. 88 . & - & - \\
\hline 66 & Fr. Eng. & 1. Nov. 88. & $\begin{array}{l}\text { 15. Jan. 91: Voll- } \\
\text { ständig. Wohlbefinden. }\end{array}$ & 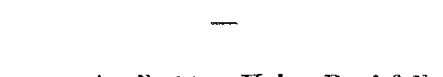 \\
\hline 67 & Fr. Weinh. & 27. Nov. 88. & - & $\begin{array}{l}\text { 4. April 91: Kein Rückfall. } \\
\text { Glatte Narbe. }\end{array}$ \\
\hline 68 & Fr. Die. $\dagger_{\text {. }}$ & 22. Dec. 88 . & - & - \\
\hline 69 & Fr. X. & 28. Jan. 89 . & 一 & $\begin{array}{l}\text { 10. Jan. 91: Kein Rückfall. } \\
\text { Glatte Narbe. }\end{array}$ \\
\hline 70 & Fr. Schö. & 7. Febr. 89 . & - & $\begin{array}{l}\text { 2. April 91: Kein Rückfall. } \\
\text { Narbe ganz weich, ohne Verhär- } \\
\text { tung. In der linken Tubenecke } \\
\text { eine hirsekorngrosse geröthete } \\
\text { Stelle. }\end{array}$ \\
\hline 71 & Fr. Ferm. †. & 19. Febr. 89. & - & - \\
\hline 72 & Fr. Scha. $\dagger$ & 11. März 89. & - & - \\
\hline 73 & Fr. Schü. & 2. April 89 & - & $\begin{array}{l}\text { 28. März 91: Keine Spur von } \\
\text { Rüclkfall. (Hausarzt.) }\end{array}$ \\
\hline 74 & Fr. Kow. & 4. April 89 & - & $\begin{array}{l}\text { 10. April 91: Kein Rückfall. } \\
\text { Glatte Narbe. }\end{array}$ \\
\hline 75 & Fr. Tip. $†$ & 11. April 89. & - & - \\
\hline 92 & Fr. Ro. †. & 15. April 89. & - & - \\
\hline 77 & Fr. Rei. & 30. April 89 . & - & $\begin{array}{l}\text { 15. März 91: Vollständiges } \\
\text { Wohlbefinden. (Hausarzt.) }\end{array}$ \\
\hline 78 & Fr. Wip. $t$. & 1. Mai 89 . & - & - \\
\hline 79 & Fr. Ullb. $\dagger$. & 7. Mai 89 . & - & - \\
\hline 80 & Fr. W. †. & 9. Mai 89 . & - & - \\
\hline
\end{tabular}


Betrachten wir aber diese 35 Todesfälle von dem uns zunächst interessirenden Gesichtspunkte der Rückfallsfrage aus, so sind 8 abzuziehen, da diese an anderen Krankheiten starben, bevor noch von dem Auftreten eines Rückfalles die Rede sein konnte. Wenn wir also diese ganz bei Seite lassen, so baben wir das Verhältniss 27 Todte : 72 Operirten $=37,5$ Proc. an Rïckfall Gestorbenen zu 62,5 Proc. nach zwei Jahren noch Lebenden. Wir haben zu den an Rückfall Gestorbenen Alle gerechnet, bei denen wir nicht mit Sicherheit eine andere Todesursache wussten.

Von den 45 noch Lebenden

$$
\begin{aligned}
& \text { haben sich vorgestellt . . . } 37=82,2 \text { Proc., } \\
& \text { gaben blos Antwort. . . . } 8=17,8 \text { " }
\end{aligned}
$$

Von den ersten 37 sind 32 bei uns in der Klinik nachuntersucht worden, 5 wurden von anderen Aerzten untersucht, theils Hausärzten, theils hier in Dresden wohnenden Gynäkologen.

Die erhaltenen Antworten lauten im allgemeinen unzweideutig, wie aus der Tabelle ersichtlich; blos eine (Nr. 30) schreibt: „Befinde mich stets unwohl"; auf' nähere Erkundigung bei dem zuständigen Arzte schreibt dieser: „Die Frau ist nach Angaben und Aussehen gesund." Während des Druckes dieser Mittheilung ist es uns noch gelungen, die Frau zu einer Vorstellung zu bewegen.

Die von uns untersuchten Frauen waren alle vollkommen gesund und fïhlten sich im allgemeinen wohl; blos bei Nr. 70 könnte man vielleicht an den allerersten Anfang eines Rückfalles denken. Fortgesetzte Beobachtung muss diese Frage noch beantworten. Nr. 30 klagte über das Harnträufeln beim Liegen aus der feinen Fistel.

Gehen wir nun zum Schlusse unsere Fälle noch einmal durch mit Berücksichtigung der bisher beobachteten Dauer der Heilung, so sind ron sämmtlichen 80 Operirten heute noch am Leben und zur Zeit gesund

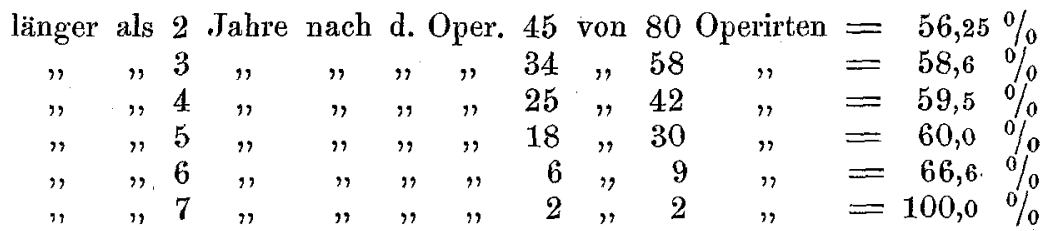

Dresden, den 5. Mai 1891. 